\title{
1 Die Homecare-Versorgung in Deutschland
}

Im Jahr 2014 waren sechs Millionen Menschen in Deutschland auf Homecare-Leistungen angewiesen - darunter Multimorbide, Ältere, aber auch Neugeborene und Kinder. Homecare steht für die Versorgung von Patienten, die entweder aufgrund einer chronischen Erkrankung auf aktive und kontinuierliche Unterstützung angewiesen sind, oder die bei einer akuten Erkrankung eine poststationäre Versorgung benötigen (BVMed, 2015b). Die Anzahl chronisch kranker, multimorbider und pflegebedürftiger Menschen nimmt kontinuierlich zu (Nowossadeck, 2012). Auch wenn diese Menschen auf eine unterstützende Betreuung angewiesen sind, möchten sie gleichzeitig möglichst lange ein selbständiges Leben in der gewohnten häuslichen Umgebung führen (Kuhlmey, Dräger, Winter \& Beikirch, 2010; Rothgang, Müller, Mundhenk \& Unger, 2014). Für Homecare bedeutet dies, dass zum Jahr 2030 ein Anstieg von über 64\% auf 9,3 Millionen Betroffene erwartet wird (BVMed, 2015a).

Eine Homecare-Therapie ist Bestandteil der ambulanten Patientenversorgung, die ärztlich angeordnet in der Häuslichkeit des Patienten bzw. im Pflege- oder Altenheim stattfindet (BVMed, 2015b). 
Patienten sind bei der Entlassung aus dem Krankenhaus meist auf sich allein gestellt, können aber gleichzeitig aufgrund ihrer chronischen Erkrankung oder gar Pflegebedürftigkeit die Weiterbehandlung nicht selbst koordinieren. Homecare schließt diese Lücke, indem es nicht nur die stationäre Versorgung mit der ambulanten Nachsorge verbindet, sondern sämtliche weitere Versorger wie Hilfsmittelhersteller, Apotheker, ambulante und stationäre Pflege oder Sozialdienste in die Koordination mit einschließt. Somit versorgt Homecare die Patienten nicht nur mit Produkten, sondern hilft bei der Koordination der ambulanten Behandlung (Reibnitz, 2009).

Homecare wird damit das Potential einer sektorenverbindenden Versorgungsform zugeschrieben, welche die schon lange angestrebte Verzahnung zwischen den einzelnen Leistungssektoren und Leistungserbringern schaffen kann (BVMed, 2015a). Inwieweit dieses Potential im Versorgungsalltag der beteiligten Leistungserbringer bereits umgesetzt wird, wurde im Rahmen einer umfassenden Versorgungsstudie untersucht. Der vorliegende Bericht zeigt die Ergebnisse der Studie in zwei Hauptteilen. Der erste Teil (Kapitel 1 bis 3) gibt einen umfassenden Überblick über die derzeitige Situation im Homecare-Bereich, die Zusammenarbeit zwischen verschiedenen Gesundheitsberufen sowie dem Entlassmanagment im deutschen Gesundheitswesen. Die Ausführungen zeigen relevante Begriffsdefinitionen auf und skizzieren rechtliche Rahmenbedingungen. Der zweite Teil umfasst die Darstellung der empirischen Studie. In Kapitel 4 werden zunächst Ergebnisse von Fokusgruppendiskussionen mit Vertretern aus Arztpraxen, Kliniken und Pflege dargestellt. Kapitel 5 beinhaltet die darauf aufbauenden Ergebnisse einer schriftlichen Befragung von Vertretern der genannten Berufsgruppen. Kapitel 6 diskutiert die gewonnenen Ergebnisse abschließend im Gesamtkontext.

Die Anzahl chronisch kranker, multimorbider und pflegebedürftiger Menschen nimmt kontinuierlich zu (Nowossadeck, 2012). Sie sind auf eine unterstützende Betreuung angewiesen, die die zahlreichen Versorgungsbereiche koordiniert und miteinander verzahnt (Reibnitz, 2009). Dabei möchte ein überwiegender Teil der Betroffenen so lange wie möglich ein möglichst unabhängiges, selbständiges Leben in der gewohnten häuslichen Umgebung führen (SVR, 2014). 


\subsection{Was ist Homecare?}

Homecare ist ein noch relativ junger Versorgungsbereich. Demnach mangelt es bislang an einer eindeutigen Definition oder gar gesetzlichen Verankerung von Homecare im deutschen Gesundheitswesen (Reibnitz, 2009).

Der BVMed definiert Homecare als

„[...] die Versorgung von Patienten mit medizinischen Hilfsmitteln, Verbandmitteln sowie medizinischer Ernährung zu Hause und in Pflege- oder Altenheimen." (BVMed, 2015b)

Die Homecare-Versorgung eines Patienten erfolgt durch geschultes Fachpersonal im Rahmen einer ärztlichen ambulanten Therapie mit dem Ziel, die Versorgungsqualität der Klinik beizubehalten. Homecare ist somit Bestandteil der ambulanten Patientenversorgung - steht jedoch für eine sektorenverbindende Versorgungsform, die dem gesundheitspolitischen Ziel „ambulant vor stationär" gerecht wird.

Homecare ist für Patienten relevant, die aufgrund einer chronischen Erkrankung auf aktive und kontinuierliche Unterstützung angewiesen sind oder die bei einer akuten Erkrankung eine poststationäre Versorgung benötigen (BVMed, 2015a). Die in der Klinik begonnene Therapie wird dann im Pflegeumfeld des Patienten weitergeführt, mit dem Ziel, das Behandlungsniveau qualitativ beizubehalten und eine erneute Einweisung in das Krankenhaus vorzubeugen (Harbord, 2009; Reibnitz, 2007).

Die Homecare-Versorgung umfasst die in Abbildung 1 beschriebenen Therapiebereiche.

Patienten sind bei der Entlassung aus dem Krankenhaus meist auf sich allein gestellt, können aber gleichzeitig aufgrund ihrer chronischen Erkrankung oder gar Pflegebedürftigkeit die Weiterbehandlung nicht selbst koordinieren. Es mangelt an Kenntnissen über alternative Therapieformen, verantwortliche Kostenträger sowie passende Einrichtungen oder Experten zur

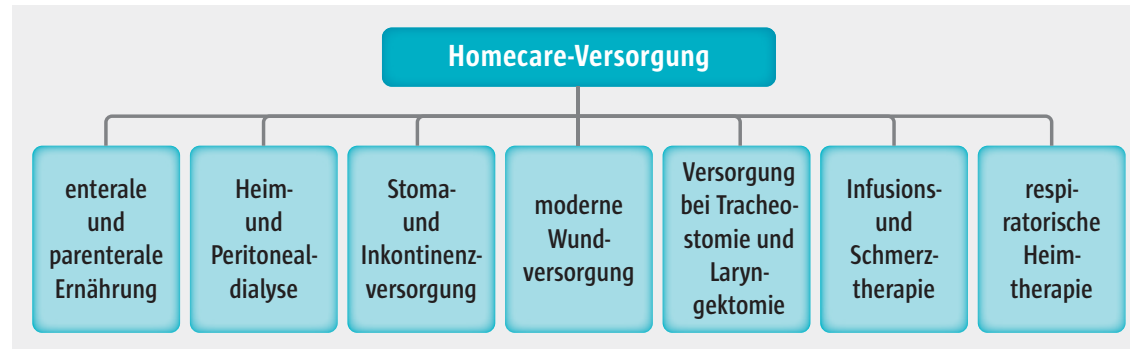

Abb. 1 Therapiebereiche der Homecare-Versorgung 


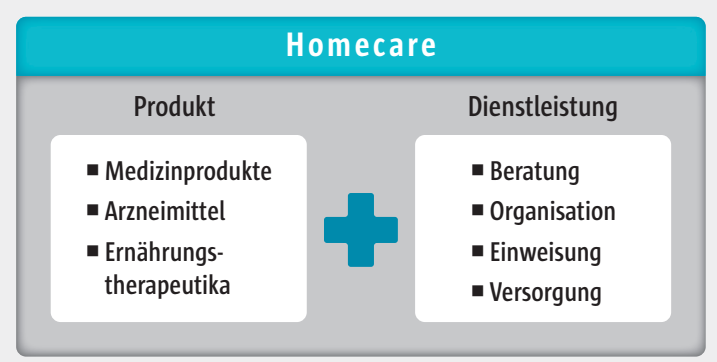

Abb. 2 Homecare als Verbindung von Produkt und Dienstleistung (eigene Darstellung in Anlehnung an Reibnitz, 2009)

Weiterversorgung. Homecare-Unternehmen schließen diese Lücke, indem sie den Patienten nicht nur mit dem Produkt versorgen und ihn dahingehend beraten und betreuen. Sie übernehmen zunehmend auch die Koordination der ambulanten Behandlung. Homecare verbindet demnach das Produkt mit der notwendigen Dienstleistung (vgl. Abbildung 2) (Reibnitz, 2009).

Eine qualifizierte Fachkraft versorgt den Patienten und dessen Betreuer mit Informationen zum Krankheitsbild und den damit verbundenen Therapieoptionen. Sie leitet den Patienten zur Selbsthilfe an und betreut ihn kontinuierlich. Dadurch wird auch der Aufbau eines Vertrauensverhältnisses möglich, welches insbesondere bei psychisch belastenden Erkrankungen an Bedeutung gewinnt (Reibnitz, 2009).

Darüber hinaus übernimmt Homecare Aufgaben wie:

- Kontaktaufnahme mit dem zuständigen Hausarzt,

- termingerechte Bereitstellung der Produkte bei Entlassung,

- Koordination mit ambulantem Pflegedienst,

- Abgleich der häuslichen Gegebenheiten mit den individuellen Patientenbedürfnissen und

- die kontinuierliche Überprüfung des Therapieplans und ggfs. Empfehlung zur Anpassung oder Produktumstellung.

Homecare-Unternehmen koordinieren die Aktivitäten sämtlicher an der Patientenversorgung beteiligter Leistungserbringer.

\subsection{Der Homecare-Versorgungsanspruch}

Homecare hat keine eigenständige Rechtsgrundlage. Allerdings ergibt sich ein Versorgungsanspruch für Versicherte im Rahmen der gesetzlichen Sozialversicherung aus unterschiedlichen Regelungen in Bezug auf die einzelnen Produkte, die Homecare umfasst (vgl. Tabelle 1). 
Tab. 1 Unterschiedliche Regelungen des Homecare-Versorgungsanspruches (eigene Darstellung in Anlehnung an Reibnitz, 2009)

\begin{tabular}{lccc} 
Verbandmittel & Arzneimittel & Diätetika & Hilfsmittel \\
$\$ 31$ I S SGBV & $\$ 31$ I S SGBV & $\$ 31$ I 2SGBV & $\$ 33$ ISGBV \\
\hline
\end{tabular}

Im Wesentlichen schließt die Homecare-Versorgung Medizinprodukte, Arzneimittel und Ernährungstherapeutika ein (Künstner, 2009). Medizinprodukte unterteilen sich in Hilfsmittel und Verbandmittel, die jeweils unterschiedlichen Regelungen der Erstattungsfähigkeit unterliegen. Bei den Ernährungstherapeutika gibt es für bestimmte Produkte Sonderregelungen in Verbindung mit der Arzneimittelrichtlinie. Arzneimittel unterliegen der komplexesten Regelung zur Erstattung und sollen daher in den folgenden Ausführungen nicht gesondert betrachtet werden. Die unterschiedlichen Regelungsgrundlagen bedingen die Komplexität des Homecare-Bereiches und dessen Gestaltung.

Unter den Leistungserbringern, die einen entsprechenden Vertrag mit der Krankenkasse haben, haben Versicherte das Recht auf freie Leistungserbringerwahl (BVMed, 2011).

Das Vertragssystem gilt seit dem 01. Januar 2010. Bis zum 01. April 2007 und einer Übergangsfrist bis zum 01. Januar 2010 galt noch die uneingeschränkte freie Leistungserbringerwahl für die Versicherten. Einzige Voraussetzung war die Zulassung als Leistungserbringer gemäß der Sozialgesetzgebung.

\subsubsection{Versorgung mit Hilfsmitteln}

Nach $₫ 33$ SGB V haben Versicherte einen Anspruch auf eine Versorgung mit Hilfsmitteln gegenüber der Krankenkasse. Der Anspruch umfasst:

- das Hilfsmittel selbst,

- Ersatzbeschaffung, Wartung und technische Kontrollen sowie

- die Ausbildung im Gebrauch des Hilfsmittels.

Hilfsmittel im Sinne des $\mathbb{3} 3$ SGB XI sind Gegenstände, die von den Leistungsempfängern getragen oder mitgeführt oder bei einem Wohnungswechsel mitgenommen werden können. Für die Einschätzung der Erforderlichkeit sind stets die Umstände des Einzelfalls maßgebend. Hilfsmittel zielen darauf $\mathrm{ab}$, einer drohenden Behinderung vorzubeugen, den Erfolg einer Heilbehandlung zu sichern oder eine Behinderung bei der Befriedigung von Crundbedürfnissen des täglichen Lebens auszugleichen. Allgemeine Gebrauchsgegenstände des täglichen Lebens sind davon ausgeschlossen (BVMed, 2011). 
Hilfsmittel sind immer sächliche Mittel wie Geräte, Gegenstände oder Apparate. Demzufolge sind sie von Heilmitteln abzugrenzen, zu denen persönliche medizinische Dienstleistungen zählen, die von nicht-ärztlichen Leistungserbringern erbracht werden, wie z.B. Physio- oder Logotherapie. Neben dem Produkt hat der Versicherte auch den Anspruch auf eine Ausbildung im Gebrauch des Hilfsmittels. In der Praxis zeigt sich jedoch, dass Krankenkassen die Kosten für solche Kurse oftmals nicht ohne weiteren Druck übernehmen.

\subsubsection{Versorgung mit Verbandmitteln und Ernährungstherapeutika}

Verbandmittel dienen der Verhütung und Versorgung von Wunden bzw. der Stabilisierung, Immobilisation, funktionalen Mobilisation bzw. Kompression von Körperteilen (Künstner, 2009). GKV-Versicherte haben nach $\mathbb{} 31$ Abs. 1 SGB V einen Anspruch auf die Versorgung mit Verbandmitteln bei ärztlicher Verordnung. Im Unterschied zu Hilfsmitteln sind Verbandmittel für den Arzt richtgrößenrelevant.

Nach $₫ 31$ Abs. 1 Satz 1-2 SGB V haben Versicherte Anspruch auf eine Versorgung mit Ernährungstherapeutika zu Lasten der Gesetzlichen Krankenversicherung (GKV). Ernährungstherapeutika dienen der Zufuhr von Nahrungssubstraten entweder unter Nutzung des verbliebenen Verdauungstrakts (enteral) oder direkt über zentral- oder periphervenöse Katheter (parenteral) (Harbord, 2009). Die Produkte gelten weder als Arzneimittel noch als Verband- oder Hilfsmittel und wurden daher ausnahmsweise als eigene Leistungskategorie in die bestehende Richtlinie zur Arzneimittelversorgung aufgenommen (Künstner, 2009). Eine ambulante Ernährungstherapie sollte grundsätzlich vom behandelnden Arzt verordnet werden.

\subsubsection{Abgrenzung zur ambulanten und stationären Pflege}

Die Homecare-Therapie ist eine Krankenbehandlung, die vom Arzt verordnet wird und speziell qualifiziertes Fachpersonal benötigt. Sie ist damit eindeutig von der (häuslichen) Pflege abzugrenzen, die neben der Behandlungsauch die Grundpflege und hauswirtschaftliche Versorgung umfasst (BMG, 2014; Reibnitz, 2009). Während die Behandlungspflege die Durchführung von medizinischen Leistungen von geschultem Fachpersonal ausschließlich nach ärztlicher Delegation beinhaltet, bedeutet Grundpflege die Versorgung eines Pflegebedürftigen beispielsweise durch die Unterstützung beim Waschen, der Mundhygiene sowie bei der Nahrungszufuhr (Reibnitz, 2009).

Bei Patienten mit Homecare-Versorgungsbedarf in Pflegeheimen stellt sich zusätzlich die Frage, ob Krankenkassen oder Pflegeheime die Kosten für die Versorgung zu tragen haben (BVMed, 2011). Die Pflegeversicherung hat gemäß \$ 69 SGB XI dafür zu sorgen, dass die Pflegebedürftigen „nach dem allgemein anerkannten Stand medizinisch-pflegerischer Erkenntnisse ausreichend und 
angemessen gepflegt werden“ ( $\$$ 69 SGB XI). Die Pflegekassen sind für die Umsetzung dessen verantwortlich und erbringen Sach- und Geldleistungen. Zu den Sachleistungen gemäß SGB XI gehört auch die Ausstattung mit Hilfsmitteln. Hierzu schließen Pflegekassen mit Trägern von Pflegeeinrichtungen Leistungsverträge $a b$, wodurch sie ihren Versorgungsauftrag auf die Pflegeheime übertragen und im Gegenzug ein Entgelt zahlen. Heimbewohner kön-

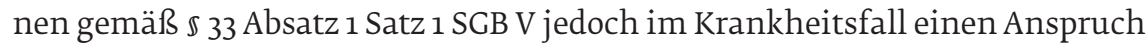
auf individuelle Versorgung mit Hilfsmitteln haben. Hier muss zwischen der Leistungspflicht der Krankenkassen und der des Pflegeheims abgegrenzt werden. Maßgebend ist, ob das Hilfsmittel für die Behandlungs- oder Grundpflege zum Einsatz kommt. Im Rahmen der Behandlungspflege ist grundsätzlich die Krankenkasse in der Leistungspflicht. Dies ist vor allem dann der Fall, wenn das Hilfsmittel medizinisch notwendig sowie individuell für den einzelnen Versicherten angepasst ist und nur für ihn bestimmt und verwendet wird. Dagegen tragen die Pflegeheime die Kosten für Hilfsmittel, die für die Grundpflege benutzt werden. Die Abgrenzung kann jedoch nicht allgemeinverbindlich oder produktspezifisch vorgenommen werden, weswegen jeder einzelne Versorgungsfall individuell zu prüfen ist (BVMed, 2011).

\subsection{Finanzierung der Homecare-Versorgung}

Kostenträger für Homecare-Leistungen ist die GKV. Bei der Erstattung von Hilfsmitteln verhandeln die Homecare-Leistungserbringer mit den einzelnen Krankenkassen Vertragspreise im Rahmen von Verträgen nach $\mathbb{} 127$ SCB V. Für sechs Produktgruppen des Hilfsmittelverzeichnisses gelten bundesweite Festbeträge. Diese Festbeträge stellen eine Obergrenze für die Erstattung von Hilfsmitteln dar. Dies bedeutet, dass die Krankenkasse den jeweils verhandelten Vertragspreis maximal bis zur Höhe des Festbetrags übernimmt (Künstner, 2009). Wenn ein Produkt ausgewählt wird, dessen Preis den Festbetrag übersteigt, hat der Versicherte den Differenzbetrag als Eigenanteil selbst zu zahlen (BVMed, 2011).

Für Verbandmittel und Ernährungstherapeutika gibt es derzeit keine Festbeträge. Die Erstattungshöhe ergibt sich hier ausschließlich aus den individuellen Verträgen zwischen Krankenkasse und Leistungserbringer (Künstner, 2009). Voraussetzungen für die Erstattung eines Hilfsmittels durch die GKV sind:

1. die Hilfsmitteleigenschaft nach dem Sozialgesetzbuch und

2. die Erforderlichkeit des Hilfsmittels im Einzelfall.

Die Hilfsmitteleigenschaft ergibt sich aus der Definition nach $\mathbb{S} 31$ SGB XI. Crundlage bildet das Hilfsmittelverzeichnis ( $\$ 139$ SGB V), das von der Leistungspflicht der GKVen eingeschlossene Hilfsmittel beinhaltet und Mindeststandards für die Produkt- und Versorgungsqualität definiert. Das Verzeichnis dient Ärzten als Orientierungshilfe, ist jedoch keine verbindliche Positivliste. 
Darüber hinaus ist die Erforderlichkeit des Hilfsmittels im Einzelfall zu prüfen. D.h., das Hilfsmittel muss geeignet und notwendig und im Vergleich zu anderen Hilfsmitteln wirtschaftlich sein, um die Behandlungsziele zu erreichen. Hierbei sind die individuellen Verhältnisse des Patienten wesentlich.

Die Krankenkasse prüft die Voraussetzungen und genehmigt die Erstattung der angeforderten Hilfsmittel auf Grundlage des durch den Leistungserbringer eingereichten Kostenvoranschlags. Kann die Krankenkasse in Einzelfällen nicht allein bewerten, ob sie die Erstattung genehmigt, schaltet sie den Medizinischen Dienst der Krankenversicherung hinzu (BVMed, 2011).

Homecare-Dienstleistungen werden allein über die Erstattung der Produktkosten finanziert (BVMed, 2015a). Insbesondere in diesem Zusammenhang sind jedoch die kontinuierlich fallenden Vergütungspreise für die Produkte kritisch zu betrachten. Langfristig wird dies zu einer Veränderung der Qualität der begleitenden Dienstleistungen führen. Vor allem stellt sich die Frage, wie viel Dienstleistung sich künftig überhaupt noch erbringen lässt und ob alternative Wege der Finanzierung denkbar sind (Künstner, 2009).

\subsection{Der Homecare-Markt in Deutschland}

Der Homecare-Markt gewinnt in Deutschland an Bedeutung. Waren im Jahr 2014 etwa sechs Millionen Menschen auf Homecare-Leistungen angewiesen, werden es bis zum Jahr 2030 Prognosen zufolge 9,3 Millionen Betroffene sein, was einem Anstieg von etwa 64\% entspricht (BVMed, 2015a). Etwa die Hälfte der Betroffenen leidet derzeit unter chronischen Wunden. Ein Zehntel benötigt aufsaugende Inkontinenzprodukte und circa $8 \%$ werden mit medizinisch enteralen Ernährungsprodukten versorgt (BVMed, 2015a).

\subsubsection{Wachstumsmarkt Homecare}

Die zunehmende Bedeutung von Homecare spiegelt sich auch im wachsenden Marktvolumen wider, das im Jahr 2013 weltweit bei circa 225 Milliarden US Dollar lag. Laut Schätzungen wird das Marktvolumen bis 2016 auf 295 Milliarden US Dollar ansteigen. Der EU-Markt für Homecare hatte im Jahr 2013 ein Volumen von 50 Milliarden US Dollar. Dies macht die EU zum zweitgrößten Markt mit einem Anteil von 23\%. Dabei zählen vor allem Deutschland, Frankreich und Großbritannien zu den Ländern mit dem größten HomecareMarkt in der EU (ConAlliance, 2014).

In Deutschland wird der Homecare-Markt auf rund 2,5 Milliarden Euro (Erstattungspreise) geschätzt und nach Prognosen in den nächsten Jahren voraussichtlich um 3 bis 5\% wachsen (Summary Seven, 2014). Den größten Anteil am Marktvolumen hat die Wundversorgung mit 31\%, gefolgt von der enteralen Ernährung mit 16\% (vgl. Abbildung 3). 


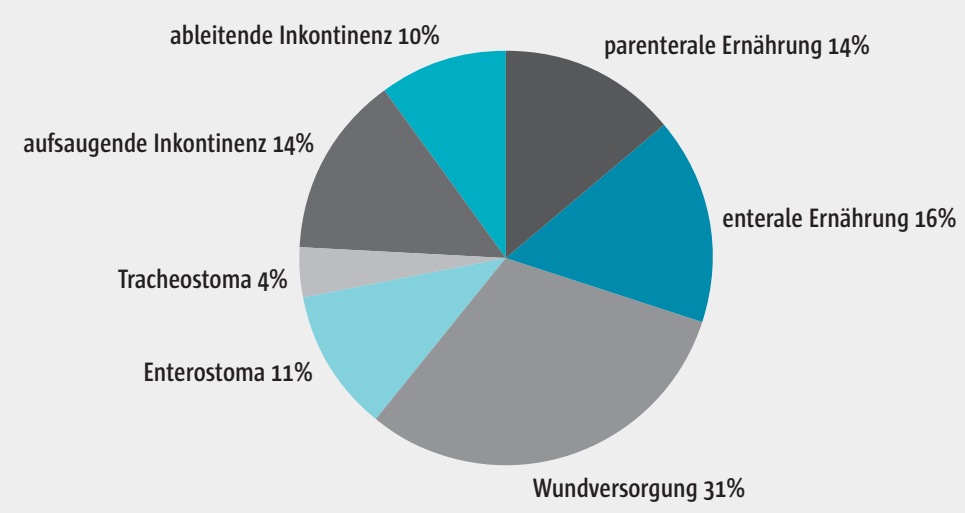

Abb. 3 Marktanteile der einzelnen Homecare-Versorgungsbereiche in Deutschland (eigene Darstellung in Anlehnung an Summary Seven, 2014)

Die Ausgabenanteile von Hilfsmitteln an den Gesamtausgaben der GKV bestätigen den Aufwärtstrend der Homecare-Branche. Zwar sind die Ausgabenanteile von Hilfsmitteln an den Gesamtausgaben mit knapp 4\% relativ gering, so wachsen sie jedoch beständig. Im Jahr 2013 stiegen die Leistungsausgaben für Hilfsmittel auf 6,8 Milliarden Euro, was einem Zuwachs von mehr als 5\% gegenüber dem Vorjahr entspricht. Bereits nach den ersten drei Quartalen 2014 zeigte sich erneut eine Erhöhung um 9,3\% (BVMed, 2015). Seit 2007 ist ein Ausgabenanstieg von über 23\% für Hilfsmittel (inklusive Hilfsmittel aus Apotheken) zu verzeichnen (vgl. Abbildung 4). Pro GKV-Versichertem gab die

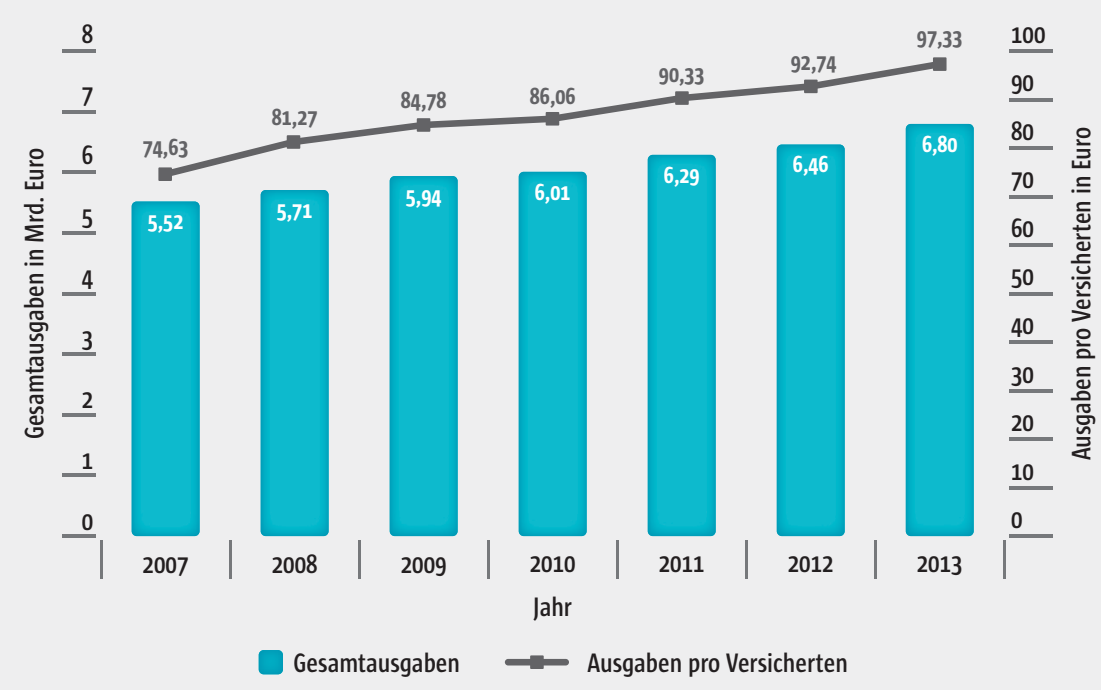

Abb. 4 Ausgaben für Hilfsmittel in der GKV - Entwicklung von 2007 bis 2013 (eigene Darstellung in Anlehnung an Sauer et al., 2014) 


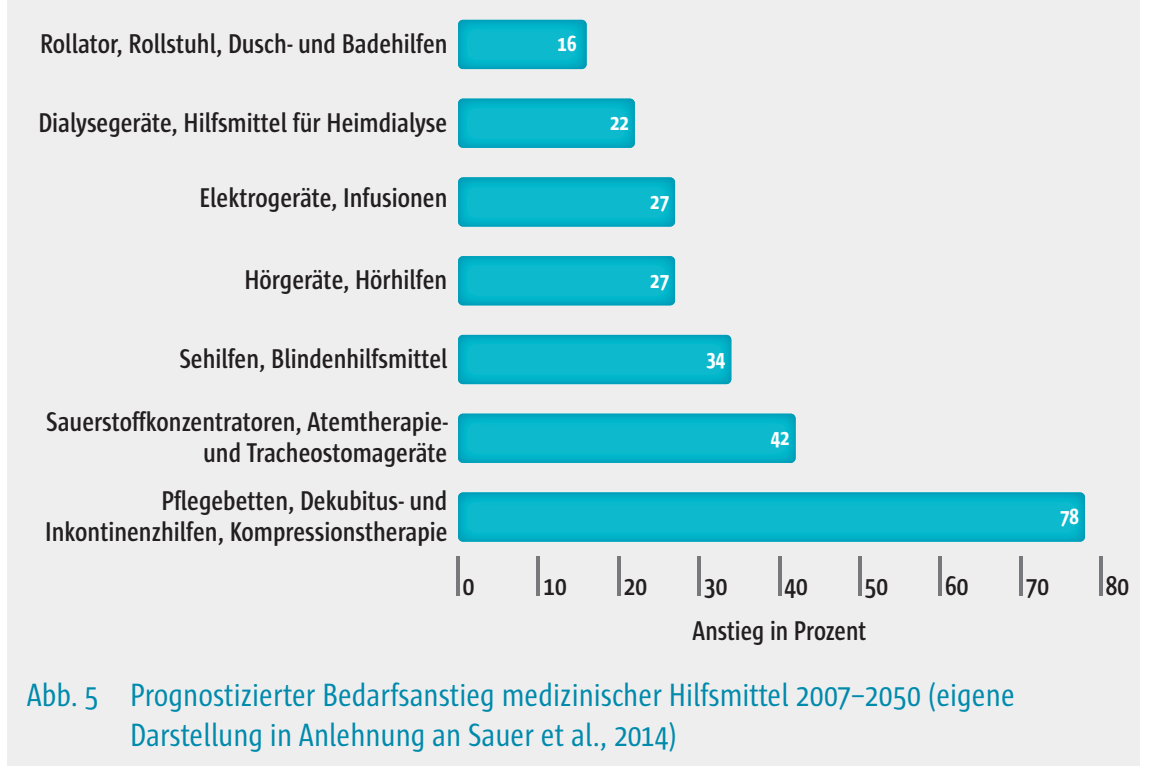

GKV im Jahr 201397 Euro für Hilfsmittel (gegenüber knapp 93 Euro im Jahr 2012) aus (Rothgang et al., 2014; Sauer et al., 2014).

Durch treibende Faktoren wie insbesondere den erwarteten demografischen Entwicklungen, dem sich verändernden Morbiditätsspektrum sowie dem medizinisch-technischen Fortschritt wird auch in der GKV mit einem weiter steigenden Bedarf an Hilfsmitteln gerechnet - bei Zuwachsraten mit bis zu $78 \%$ (vgl. Abbildung 5).

Das künftige Wachstum ist so betrachtet jedoch in erheblichem Umfang von der Entwicklung der Erstattungspreise abhängig (Sauer et al., 2014). Gemäß Branchenprognosen wird der Versorgungsbereich der enteralen Ernährung geprägt von Mengenausweitungen und Preisreduktionen - durchschnittlich um $4 \%$ jährlich sinken. Hingegen wird dem Bereich der Wundversorgung ein großes Wachstumspotential zugeschrieben, da die Erstattungspreise der Kassen für moderne Wundversorgungsprodukte kontinuierlich steigen und noch etwa zwei Drittel der Patienten klassisch versorgt werden (Summary Seven, 2014).

\subsubsection{Wachstumstreibende Faktoren}

\section{Veränderung des Alters- und Krankheitsspektrums}

Ein langes Leben wird immer häufiger zur gesellschaftlichen Realität. Während im Jahr 2012 noch circa 20,5 Millionen Menschen in Deutschland über 
60 Jahre alt waren, werden es im Jahr 2050 schon mehr als 25 Millionen und somit etwa 38\% der Gesamtbevölkerung sein. Besonders deutlich zeigt sich die Alterung in der Anzahl der Hochbetagten. So wuchs die Gruppe der 85- bis 90-Jährigen im Zeitraum von 1952 bis 2008 um etwa 795\%, die der über 90-Jährigen sogar um $1.860 \%$ (Schaeffer \& Kuhlmey, 2012).

Für den Homecare-Markt ist in diesem Zusammenhang interessant, dass die Versorgung von Versicherten mit Hilfsmitteln fast linear mit dem Alter zusammenhängt. Mit Ausnahme der Altersgruppen bis unter 20 Jahren steigt der Anteil derer mit Bedarf an Hilfsmitteln mit dem Alter von 10\% auf 69\% (vgl. Abbildung 6) (Sauer et al., 2014).

Auch wenn die Frage, ob die steigende Lebenserwartung mit einem Mehr an gesunden oder an kranken Lebensjahren verbunden sein wird, nach wie vor zur Diskussion steht, steigt zumindest die Wahrscheinlichkeit von Krankheit und Beeinträchtigung im hohen Alter zweifelsfrei. Dabei ändert sich im Zuge des Altersstrukturwandels auch das Krankheitsspektrum. Chronische Erkrankungen machen heute rund 46\% der gesamten weltweiten Krankheitslast aus (Schaeffer \& Kuhlmey, 2012). Ab einem Alter von 65 Jahren weisen mehr als die Hälfte aller Menschen mindestens eine chronische Erkrankung auf (Nowossadeck, 2012). Dieser zunehmende Trend der chronischen Erkrankungen wird sich künftig auch weiter fortsetzen (vgl. Abbildung 7).

Neben chronischen Erkrankungen steigt mit dem Alter auch die Wahrscheinlichkeit von Multimorbidität sowie Pflegebedürftigkeit. Diese Korrelation zeigt eindrücklich die Notwendigkeit neuer Versorgungskonzepte im Gesundheitswesen, die den künftigen Entwicklungen entgegen kommen.

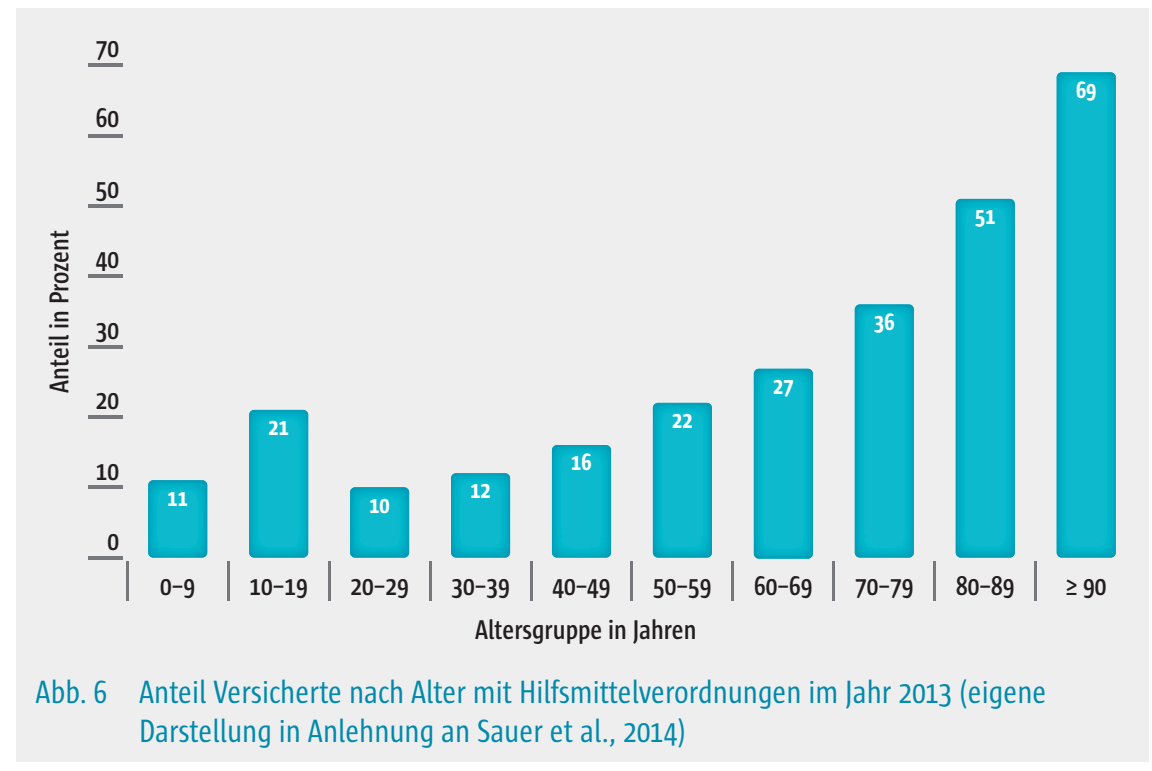




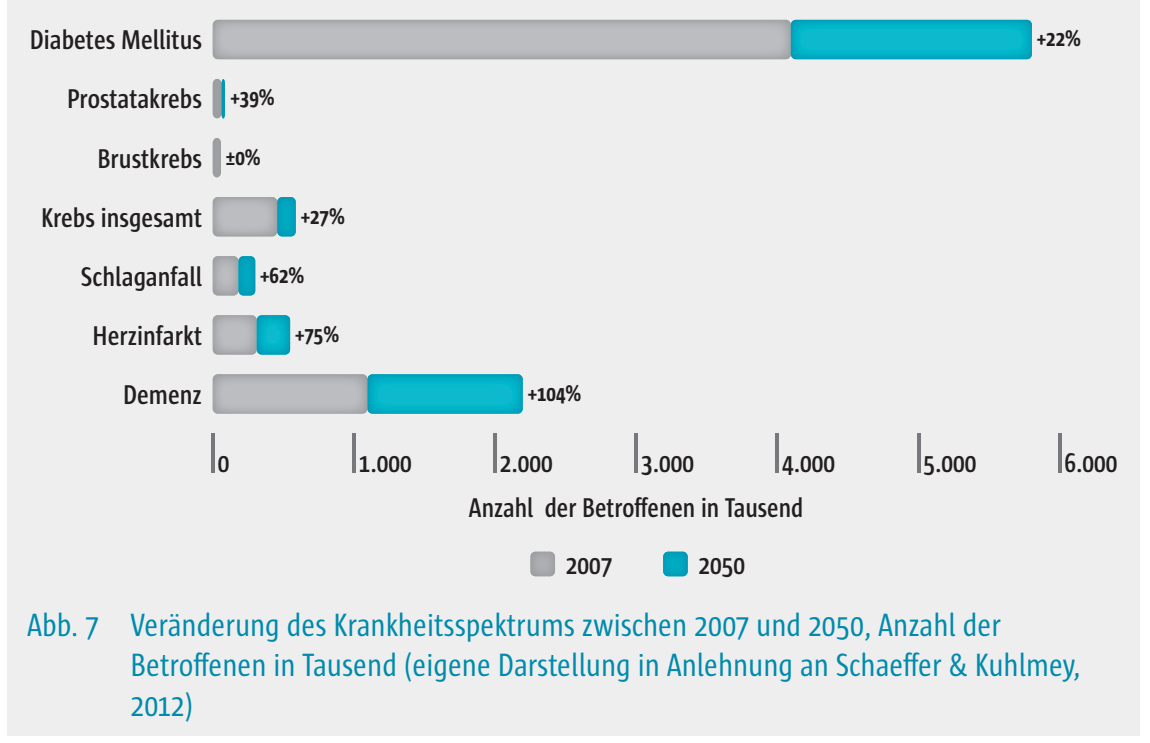

Dies betrifft insbesondere auch den Homecare-Bereich, da gerade chronisch kranke, multimorbide Pflegebedürftige einen wachsenden Bedarf an Homecare haben.

\section{Technischer Fortschritt und das Zuhause als Gesundheitsstandort}

Mit der Zunahme an Lebensjahren, die mit gesundheitlicher Einschränkung oder Pflegebedürftigkeit verbracht werden, steigt auch der Wunsch nach einem möglichst langen Verbleib in der eigenen Häuslichkeit. Einer Studie im Jahr 2010 zufolge wünschen sich 40\% der befragten Männer und Frauen die Pflege durch Angehörige und ambulante Pflegedienste. Insgesamt bevorzugen 64\% aller Befragten eine Versorgung in der eigenen Häuslichkeit durch Angehörige und ambulante Pflegedienste (vgl. Abbildung 8).

Auch hier ist ein steigender Trend in den kommenden Jahren anzunehmen. Das Zuhause wird somit mehr und mehr zum Gesundheitsstandort. Möglich wird dies im Wesentlichen durch den medizinisch-technischen Fortschritt, der die Struktur und Art der ärztlichen Leistungserbringung zum Teil grundlegend verändert. So ermöglichen neue Therapieverfahren, dass Patienten frühzeitiger aus dem Krankenhaus entlassen und im Rahmen der Homecare-Versorgung weitertherapiert werden können. Sowohl vereinfachte als auch völlig neue Therapieformen sind heutzutage im ambulanten, häuslichen Bereich möglich (Reibnitz, 2009). Beispielsweise können heute Patienten in ihrem Zuhause beatmet werden oder eine künstliche Ernährung erhalten. 


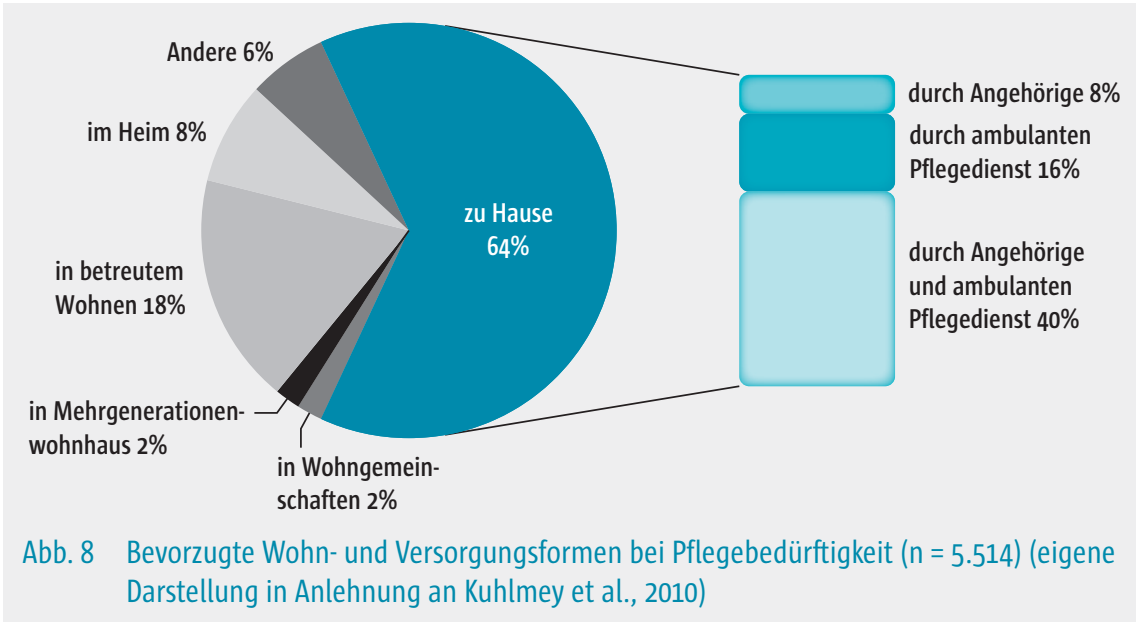

\section{DRG-Erlöslogik führt zu kürzeren stationären Verweildauern}

Der Trend zur „Ambulantisierung“ der Versorgung setzte bereits mit der Einführung des DRG-Entgeltsystems im stationären Sektor ein. Wie Abbildung 9 verdeutlicht, ergibt sich nur beim Unterschreiten der durchschnittlichen Verweildauer ein positiver Deckungsbeitrag für das Krankenhaus. Sobald

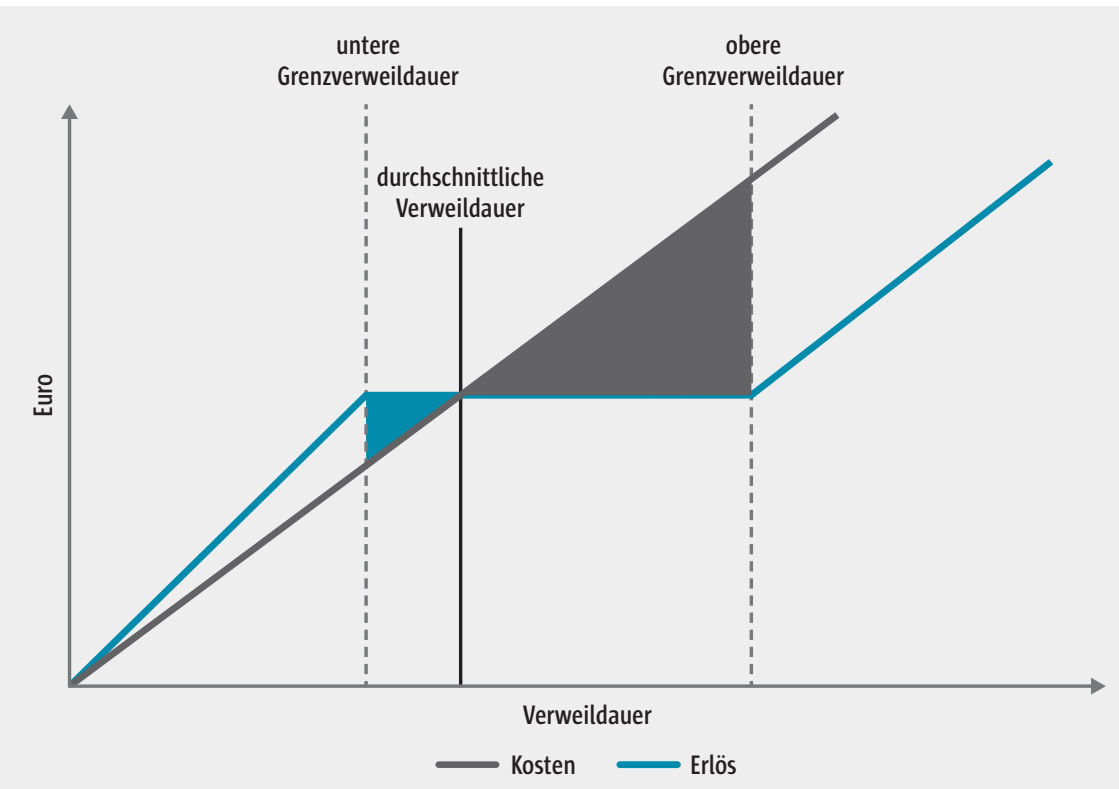

Abb. 9 Erlössituation von Krankenhäusern im DRG-System in Abhängigkeit der Verweildauer (eigene Darstellung in Anlehnung an Flux, 2010) 
ein Patient länger als der Durschnitt stationär versorgt wird, übersteigen die Kosten den durch Abschläge reduzierten DRG-Erlös.

Krankenhäuser sehen sich nach der DRG-Logik somit veranlasst, die Verweildauer von Patienten weitestgehend zu reduzieren. Dies zeigt sich auch bedeutend in den Statistiken seit Einführung des DRG-Systems. In den Jahren 2000 bis 2012 sank die durchschnittliche Verweildauer von 9,7 Tage auf 7,6 Tage, ohne dass die Patienten zwangsläufig schneller vollständig genesen wären (Schelhase, 2007, 2014). Patienten werden demnach in einem früheren Genesungsstadium entlassen und sind zurück in der eigenen Häuslichkeit zumeist auf sich gestellt und mit der veränderten Situation überfordert. Die Wahrscheinlichkeit einer Wiedereinweisung des Patienten ins Krankenhaus innerhalb kurzer Zeit ist nicht zu unterschätzen. Eine adäquate medizinische Nachsorge hingegen könnte einer Wiedereinweisung vorbeugen. Homecare ermöglicht in diesem Zusammenhang die Versorgung des Patienten mit indikationsgerechten Produkten, berät, koordiniert und gibt Anleitung zur Selbsthilfe.

Die hier dargestellten treibenden Faktoren lassen das Wachstumspotential der Homecare-Branche mehr als nur erahnen. Angesichts des zu erwartenden Bedarfszuwachses an ambulanter, häuslicher Betreuung kann Homecare in Zukunft eine vollkommen neue Bedeutung zugesprochen werden.

\subsubsection{Unternehmensstrukturen im Homecare-Bereich}

Der Hilfsmittelmarkt wird für Hersteller und Anbieter stetig lukrativer. Entwicklungen in diesem Bereich sind technisch hoch differenziert und innovativ (Sauer et al., 2014). Entsprechend haben sich die Unternehmensstrukturen im Homecare-Markt in den letzten Jahren deutlich verändert.

Während lokale Fachhändler mit einem Schwerpunkt auf Homecare anfangs den Markt dominierten, sind heute zunehmend Unternehmen ausschließlich auf Homecare spezialisiert (Summary Seven, 2014). Circa 30\% des gesamten Markts werden von fünf überregional tätigen Anbietern abgedeckt. Neben diesen großen Homecare-Spezialisten gibt es circa 100 regional fokussierte Homecare-Anbieter sowie weitere 500 Sanitätsfachhändler mit einer eigenen Homecare-Fachabteilung, die ebenfalls zusammen einen Marktanteil von circa 30\% ausmachen. Der restliche Markt verteilt sich auf Apotheken und Sanitätsfachhändler ohne Homecare-Angebot, die in den letzten fünf Jahren an die Homecare-Spezialisten abgegeben haben, deren Umsatzzahlen zeitgleich kontinuierlich stiegen. Tendenziell wird sich der Markt durch Fusionen und Übernahmen weiter verdichten, sodass wenige große Homecare-Anbieter marktbeherrschend bleiben.

Einige dieser Homecare-Spezialisten beteiligen sich vermehrt auch direkt an der Patientenversorgung. Sie bilden eigene Mitarbeiter, sog. Case-Manager, für die Homecare-Versorgung aus. Sie übernehmen nicht nur die Produktver- 
sorgung sondern ebenfalls Behandlungsleistungen (Harbord, 2009). Zudem koordinieren und dokumentieren sie den Behandlungsablauf und weisen den Patienten in die Geräte und Hilfsmittel ein (Harbord, 2009).

\subsection{Homecare als Versorgungsherausforderung}

\subsubsection{Homecare als Spezialisierung}

Nicht nur, aber gerade auch im Homecare-Bereich stellt sich die Frage, wie viel Spezialisierung es in der Medizin bedarf. Während eine generalistische angelegte Herangehensweise den Vorteil bringt, den Patienten im Gesamtbild seiner körperlichen Konstitution zu betrachten, ermöglicht Spezialisierung eine indikationsspezifisch differenzierte und kompetente Betreuung. Der Spezialist hat dann den Vorteil über eine große Menge an Fällen in seinem Spezialgebiet einen entsprechenden Erfahrungsschatz aufzubauen und von Lernkurveneffekten zu profitieren.

Die Frage nach dem Zusammenhang zwischen Menge und Qualität besteht bereits seit längerem in der Gesundheitsversorgung. Dahinter stellt sich die These auf, dass Ärzte und Krankenhäuser mit größerem Behandlungsvolumen an bestimmten Eingriffen oder Prozeduren bessere Ergebnisse in diesem Bereich erzielen als andere.

In Deutschland hat die Qualitäts-Mengen-Debatte über die Mindestmengenregelung nach $\mathbb{} 137$ SGB V Eingang in die Gesundheitsversorgung gefunden. Mindestmengen wurden erstmals im Jahre 2003 für Leber-, Nieren- und Stammzellentransplantation sowie für Ösophagus- und Pankreaseingriffe vereinbart (Velasco-Garrido \& Busse, 2004). Der Katalog wird kontinuierlich um zusätzliche Indikationen erweitert (IQWiG, 2006).

Die Empirie zeigt, dass es insbesondere im operativen Bereich für hochrisikoreiche, aber seltene Prozeduren Unterschiede in den Patienten-Outcomes in Abhängigkeit der erbrachten Menge gibt, speziell in Bezug auf die postoperative Mortalität (Pietsch et al., 2013).

Im Homecare-Bereich besteht bislang keine Evidenz über die Relevanz von Mindestmengen für eine qualitativ hochwertige Versorgung. Allerdings gehören die komplexen Krankheitsbilder der Homecare-Patienten nicht zwangsläufig zum typischen Patientenprofil einer Hausarztpraxis und machen meist nur einen kleinen Prozentteil aus (Nielandt, 2009). Daneben ist das Angebot an Hilfsmitteln und anderen Homecare-Produkten sehr heterogen und umfassend. Auf dem deutschen Markt sind allein mehr als 30.000 Hilfsmittel verfügbar. Eine adäquate Homecare-Versorgung setzt demnach den Einsatz von spezifischem Fachpersonal voraus, das in den typischen Homecare-Therapien, die in den Curricula der Crundausbildungen nicht bzw. unvollständig integriert sind, geschult wird (Nussbaumer, 2009). 


\subsubsection{Der idealtypische Homecare-Versorgungsprozess}

Im idealtypischen Homecare-Versorgungsprozess steht der HomecareDienstleister dem Patienten und dessen Angehörigen als Hilfsmittelspezialist und Dienstleister zur Seite (vgl. Abbildung 10). Er koordiniert das qualitätsgesicherte Überleit- und Entlassmanagement aus dem Krankenhaus in den ambulanten Bereich und ist Therapiespezialist zur Sicherstellung der ambulanten Therapie und Vermeidung von Komplikationen sowie Rehospitalisierungen (Döring, 2014).

Die ambulante Weiterbehandlung beginnt in der Regel mit einem vom niedergelassenen Arzt ausgestellten Rezept. Ärzte verordnen dabei nur die Produktart. Der Homecare-Fachhandel (z.B. Sanitätshaus) wählt das Einzelprodukt aus und trägt damit auch die Verantwortung für die Auswahl und Abgabe des wirtschaftlich günstigsten Hilfsmittels (BVMed, 2011).

Am häufigsten entsteht der Homecare-Versorgungsbedarf bei Patienten, die nach einem Krankenhausaufenthalt aus der Klinik entlassen werden. Der koordinierte Übergang des Patienten in den ambulanten Versorgungssektor ist von entscheidender Bedeutung für den weiteren Genesungsverlauf und erfordert ein entsprechendes Überleit- und Entlassmanagement von Seiten der Klinik. Dazu meldet die Klinik den Patienten rechtzeitig vor Entlassung und plant gemeinsam mit dem Homecare-Unternehmen die unmittelbare poststationäre Versorgung. Das Homecare-Unternehmen stellt einen spezialisierten Ansprechpartner, der sich mit dem Entlassmanagement, dem Sozialdienst, den Klinikärzten und den Pflegemitarbeitern abstimmt. Er klärt den Patienten und die Angehörigen noch während des Klinikaufenthaltes über den weiteren Verlauf der Therapie auf und stellt Kontakt zum ambulant weiterbehandelnden Arzt sowie der weiterversorgenden Pflegeeinrichtung bzw. dem ambulanten Pflegedienst her. Gleichzeitig dokumentiert

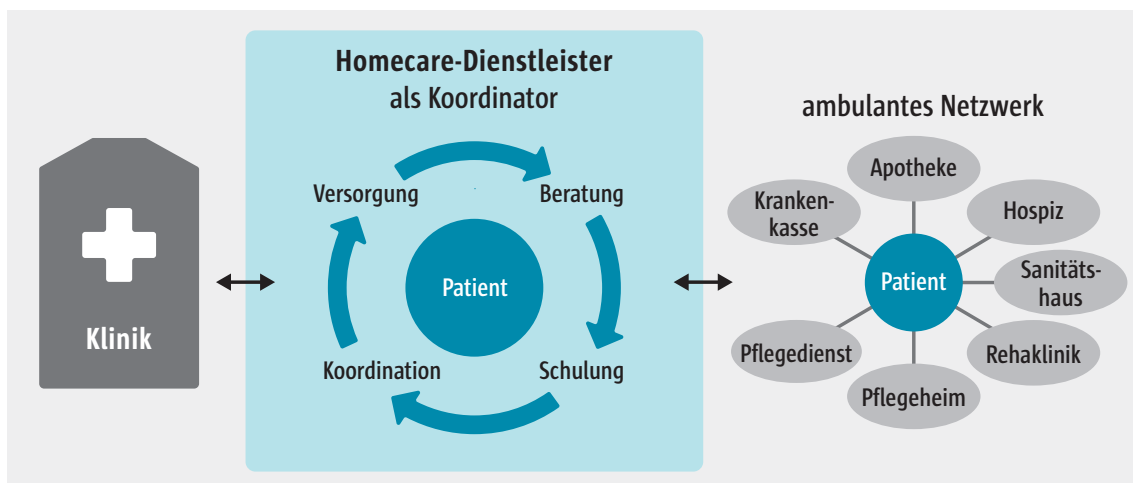

Abb. 10 Der Homecare-Dienstleister als Koordinator (eigene Darstellung in Anlehnung an Döring, 2014) 
der Homecare-Manager alle notwendigen Daten und pflegerischen Informationen zu Diagnosen, Behandlungen und Weiterbehandlungsvorschlägen.

Die Qualifikation des Homecare-Managers ermöglicht es ihm, die Produkte herstellerunabhängig sowie sinnvoll auszuwählen und kann dabei auch das Wirtschaftlichkeitsgebot des Hausarztes berücksichtigen. Der HomecareManager leitet rechtzeitig alle administrativ erforderlichen Vorkehrungen (insbesondere ärztliche Verordnungen) ein, um die Kostenübernahme der Krankenkasse zu klären und die notwendigen Hilfsmittel rechtzeitig zum Zeitpunkt der Entlassung vor Ort beim Patienten zu haben.

Vor Ort im Pflegeumfeld des Patienten weist der Homecare-Manager Pflegemitarbeiter, Patienten sowie Angehörigen in die Anwendung der Produkte ein und steht als Experte jederzeit als Ansprechpartner zur Verfügung. Er führt regelmäßige Therapievisiten durch und dokumentiert Verlauf sowie Fortschritt der Versorgung für den behandelnden Arzt und die Krankenkasse. Er ist Ansprechpartner bei Versorgungsproblemen und Therapieumstellungen und bindet vorhandene Netzwerke (Logopäden, Seelsorge etc.) ein. Alle beteiligten Leistungserbringer können auf die relevanten Informationen über Behandlungsverlauf, verursachte Kosten und Qualität der Behandlungspflege mittels einer elektronischen Fallakte zugreifen. Die fallbezogene Dokumentation erlaubt auch eine gemeinschaftliche Evaluation der Versorgungsqualität.

\subsubsection{Herausforderungen einer bedarfsgerechten Homecare-Versorgung}

Den idealtypischen Versorgungsprozess findet man in der Praxis nur selten vor. Es fehlen einheitliche Versorgungsstrukturen, Qualitätsvorgaben und Qualitätskontrollen. Zudem ist der Homecare-Versorgungsprozess durch eine Vielzahl an beteiligten Stakeholdern gekennzeichnet, die alle unterschiedlichen Regelungsbereichen und Versorgungslogiken unterliegen und auf ein sehr heterogenes Feld an Unternehmensstrukturen im Homecare-Markt treffen. Durch eine fehlende Definition und Kontrolle der Produkt- und Dienstleistungsqualität hat jedes Unternehmen sein eigenes Verständnis vom optimalen Versorgungsprozess. Die Versorgung erfolgt zudem überwiegend produkt- und einzeltherapieorientiert. Die verschiedenen Qualitätsstandards führen zu unterschiedlichen Versorgungsniveaus, die eine flächendeckende sowie qualitätsgesicherte Homecare-Versorgung infrage stellen (Döring, 2014).

In der Konsequenz kommt es insbesondere an den Schnittstellen der einzelnen Leistungssektoren (vgl. Abbildung 11) immer wieder zu Versorgungsbrüchen aufgrund von Koordinations- und Kommunikationsdefiziten.

Schnittstelle Krankenhaus: Der koordinierte Übergang des Patienten in den ambulanten Versorgungssektor ist von entscheidender Bedeutung für den weiteren 


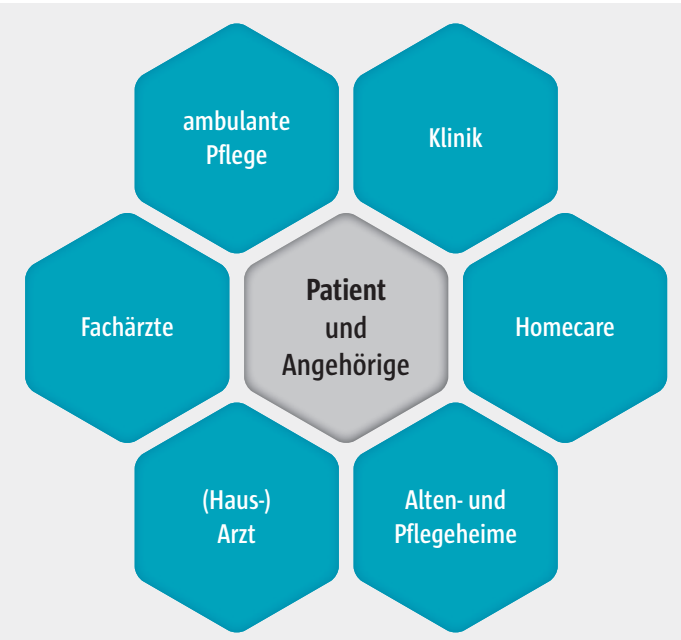

Abb. 11 Beteiligte Akteure und Schnittstellen in der Homecare-Versorgung

Genesungsverlauf und erfordert ein entsprechendes Überleit- und Entlassmanagement aufseiten der Klinik. Der Expertenstandard „Entlassmanagement" ist bislang jedoch nur unzureichend in der Praxis umgesetzt. Die Kliniken definieren vorrangig individuelle Vorgaben, wodurch die notwendige Abstimmung mit den ambulanten Versorgungspartnern bei der Entlassung häufig nur unzureichend bzw. erst nach der Entlassung erfolgt (Döring, 2014). Zwar gibt es Überleitungsberichte, die den reibungslosen Übergang des Patienten ermöglichen sollen. In der Realität zeigt sich jedoch, dass die darin enthaltenen Informationen oftmals nicht aktuell bzw. unvollständig sind. Verordnungen werden unzureichend bzw. uneinheitlich ausgestellt und der Verlegungszeitpunkt ist im Vorfeld häufig unklar oder wird kurzfristig verschoben - nicht selten eher aus Erlös- und Bettenauslastungsgründen als aufgrund des Zustands des Patienten (Grap, 2014).

Schnittstelle weiterbehandelnder Arzt und Therapeuten: Patienten werden häufig vor dem Wochenende entlassen, wobei dann kein weiterbehandelnder Arzt unmittelbar für den Patienten zur Verfügung steht. Eine Institution der Rufbereitschaft fehlt in diesem Fall. Auch in Bezug auf sonstige weiterbehandelnde Therapeuten mangelt es eindeutig an verantwortlichen Ansprechpartnern für die Patienten (Grap, 2014).

Schnittstelle Hilfsmittellieferant: Die Hilfsmittelbedarfsfeststellung orientiert sich bislang vorrangig an der einzelnen Produktversorgung und weniger am gesamten Versorgungsprozess. Gerade bei chronisch erkrankten und multimorbiden Patienten ist jedoch eine ganzheitliche Betrachtung und Versorgung des Patienten entscheidend. Zudem wird der Bedarf teilweise unvollständig erfasst und erfolgt häufig zu spät, sodass der Patient zum Zeitpunkt 
der Entlassung noch nicht ausreichend mit den notwendigen Produkten versorgt ist (Döring, 2014).

Versorger werden of tmals schon im Vorfeld der Entlassung beauftragt, sodass Patienten ihr Wahlrecht nur eingeschränkt wahrnehmen können. Da es aber gleichzeitig auch an einer umfassenden Aufklärung im Vorfeld der Homecare-Therapie mangelt, sind Patienten und Angehörige oftmals ohnehin mit der Entscheidungsfindung überfordert (Grap, 2014).

Schnittstelle Krankenkasse: Durch den häufig ungewissen Termin besteht bei der Entlassung meist noch keine Kostenzusage der Krankenkasse für die ambulante Weiterversorgung mit Hilfsmitteln. Der hohe bürokratische Aufwand führt zur weiteren Verzögerung bei der Prüfung der Verordnungen, insbesondere bei Unklarheiten durch unvollständige oder uneinheitliche Verordnungspraktiken der Krankenhäuser. Teilweise müssen Verordnungen gegenüber der Krankenkasse gerechtfertigt werden, was bei einer unzureichenden Dokumentation der zugrunde liegenden Diagnosen und Begründungen schwierig ist. Dabei gehen Homecare-Unternehmen zumeist in Vorleistung, um die Zeit bis zur Kostenzusage zu überbrücken. Für Patienten und Angehörige stellt die Ungewissheit durch die zeitlichen Verzögerungen eine zusätzliche Belastung dar (Grap, 2014).

\subsubsection{Perspektiven für die Homecare-Versorgung}

Für künftige Versorgungsnetzwerke wird die Prozessqualität und damit vor allem die Dienstleistungsqualität an Bedeutung gewinnen. Dabei werden Vergütungsregelungen neben Produkt- und Dienstleistung auch den Nutzen für den Gesamtversorgungsprozess berücksichtigen, wodurch indikationsorientierte Ansätze an Bedeutung gewinnen. Hierfür benötigt Homecare jedoch vermehrt Fachkräfte, die als Case-Manager den Patienten durch Versorgung begleiten (Döring, 2014).

Die Vernetzung zwischen den Versorgungspartnern - auch digital - wird zunehmen im Zuge eines Wettbewerbs um die erreichte Ergebnisqualität im Gesamtversorgungsprozess werden. Erfolgsentscheidend scheint dabei die Erfüllung der unterschiedlichen Kundenbedürfnisse (Patient, Arzt, Krankenhaus), klare Aufgabenzuweisungen im Versorgungsnetzwerk, Qualitätssicherung durch ambulante Behandlungspfade sowie die Dokumentation, Messbarkeit und Kontrolle der Ergebnisqualität (Döring, 2014). 